\title{
Coexistence of salmonella typhi associated hepatitis and pancreatitis in a South Indian male-a case report
}

\begin{abstract}
Enteric fever is predominantly a tropical disease caused by Salmonella typhi and paratyphi. Enteric fever commonly presents with altered liver function tests, but Salmonella is a rare cause for acute hepatitis presentation. Also Salmonella lead to acute pancreatitis with rhabdomyolysis leading to renal failure. We report an otherwise immunocompetent adult male who presented with acute hepatitis with coagulopathy, acute pancreatitis along with rhabdomyolysis, renal failure and delirium which finally turned out to be a case of Salmonella typhi causing enteric fever.
\end{abstract}

Keywords: salmonella typhi, hepatitis, pancreatitis, enteric fever, rhabdomyolysis, paratyphi, coagulopathy, abdominal pain, interstitial pancreatitis, acute hepatitis, tenderness, typhoid, renal failure, male, salmonella pancreatitis

\author{
Volume 3 Issue 3 - 2015
}

\author{
Prasanth Thayyil Sudheendran, Chethan \\ Govindaraju, Paul Cheruvathur, Premaletha \\ Narayanan, Kattoor Ramakrishnan, Vinaya \\ Kumar \\ Department of Medical Gastroenterology, Government Medical \\ College, India
}

\begin{abstract}
Correspondence: Prasanth Thayyil Sudheendran, Department of Medical Gastroenterology, Government Medical College, TC/I344(3), Koottamvila Road, Vettamukku, Vattiyorkavu P O, Thiruvananthapuram 6950 I3, Kerala, India, Tel 047I 236446I, 9446190580, Email prasanthts 1984@gmail.com
\end{abstract}

Received: September 0I, 2015 | Published: November 20,

2015

\section{Introduction}

Enteric fever is predominantly a tropical disease caused by Salmonella typhi and paratyphi. The incidence of Enteric fever in India comes to around 980 cases per 1,00,000 per year. ${ }^{1}$ Salmonella infection leads to diffuse organ involvement of liver, kidneys, heart, intestine, skin, muscle, nervous system and pancreas. Enteric fever commonly presents with altered liver function tests, but Salmonella is a rare cause for acute hepatitis presentation. Also Salmonella lead to acute pancreatitis with rhabdomyolysis leading to renal failure. ${ }^{2}$ These are the uncommon presentations of a common disease in our part of the world. Salmonella pancreatitis ${ }^{3}$ will not respond to usual line of conservative management, but need specific antimicrobial treatment in addition to aggressive supportive management. We report an otherwise immunocompetent adult male who presented with acute hepatitis with coagulopathy, acute pancreatitis along with rhabdomyolysis, renal failure and delirium which finally turned out to be a case of Salmonella typhi causing enteric fever.

\section{Case report}

A previously healthy 27 year old male belonging to a low socioeconomic class; social drinker with history of recent travel and stay at a distant place, was admitted with history of severe abdominal pain suggestive of pancreatitis of 5days duration along with non bilious vomiting and multiple episodes of loose stools without blood. $\mathrm{He}$ also reported to have severe myalgia along with low grade fever and anorexia. No h/o recent ethanol intake or toxins, but recent intake of paracetamol of $1500 \mathrm{mg} /$ day for 3days along with amoxicillin and pantoprazole for 3 days prior to admission.

On examination, he was delirious, dehydrated, mildly icteric with coated tongue with pulse rate of 102 beats per minute with Blood pressure of around $120 / 70 \mathrm{~mm} \mathrm{Hg}$ with axillary temperature of $100 \mathrm{~F}$. Abdominal examination showed enlarged liver $2 \mathrm{~cm}$ below costal margin with traube's space dull and having diffuse abdominal tenderness with no rebound tenderness. On evaluation, he had bicytopenia initially and later pancytopenia with liver function tests showing acute hepatitis picture with SGOT/PT 417/1265 with PT INR 1.75 with Amylase Lipase more than 3times the upper limit of normal(ULN) (>1000). He also had Creatine Phosphokinase 4182 (>5times ULN) with urine myoglobin positive. He also had pre renal failure (Urea 150 Creatinine 2.3) with LDH 1772 with ALT: LDH ratio 0.7 .

Serological investigations for acute hepatitis were negative for Hepatitis A, B, C E, HSV, CMV, EBV, leptospira, Dengue and Scrub were negative, but Widal came as positive 1:400 for $\mathrm{O}$ and $\mathrm{H}$ antigens. In the meantime on the 3 rd day blood culture came positive for Salmonella typhi with urine and stool culture showing no growth. Radiographic examination of Abdomen showed no evidence of perforation with USG abdomen showed mild hepatosplenomegaly with bulky pancreas. CT scan of abdomen showed acute interstitial pancreatitis with no local complications. CT scan of brain and CSF study were normal. Bone marrow study showed evidence of bone marrow suppression. Initially the patient was started on Inj.Piperacillin Tazobactam but he continued to be febrile. After getting blood culture reports, he was started on Inj.Ceftriaxone and Inj.Ciprofloxacin (sensitive antibiotics) and after 3 days he became afebrile and made dramatic improvement. On discharge, he was fully conscious oriented, ambulant, hematological and biochemical parameters almost normalized.

\section{Discussion}

Salmonella typhi causing enteric fever is a major cause for fever, abdominal pain with altered liver function tests. But Salmonella typhi causing acute hepatitis picture with coagulopathy is a rare presentation. William Osler ${ }^{4}$ has given an early insight into the hepatic manifestation of typhoid fever. Hepatomegaly with moderate transaminitis in more than $60 \%$ of cases but only $9.7 \%$ with prolonged PT INR. ${ }^{5}$ The documented incidence of Salmonella hepatitis in studies from Thailand varies from $<1 \%$ to $26 \%$ of patients. ${ }^{6}$ The probable 
associated factors for enteric hepatitis are virulence of the organism, delayed treatment and poor health of patients. ${ }^{6}$ In our patient he did not receive adequate treatment for 1 week and he continued strenuous activity in spite of symptoms as he was staying alone in distant place. The proposed mechanisms for Salmonella hepatitis are multifactorial like endotoxin, local inflammatory and or host immune reaction. ${ }^{6}$

The peak liver function test value for reported cases were bilirubin $3.6 \mathrm{mg} / \mathrm{dl}$ with AST $792 \mathrm{IU}$ and ALT 1247 with ALT:LDH ratio <9 similar to that of our case. ${ }^{7}$ The interesting fact is that most of the reported cases of rare complications are among young adults, similar to that of our case, probably because they did not receive adequate treatment or due to robust immune reaction. ${ }^{2,3}$ There are also case reports of coexistence of enteric fever with Viral hepatitis A, but have ALT: LDH ratio more than 9, but in our case ALT: LDH ratio $<9$ and IgM HAV negative. ${ }^{8,9}$ Salmonella pancreatitis is also a rarely reported complication with incidence not clearly reported in literature. Our patient had amylase lipase $>3$ times ULN with abdominal pain suggestive of pancreatitis and $\mathrm{CT}$ scan abdomen showing acute interstitial pancreatitis. In a case report from Coimbatore Kadappu et al., ${ }^{3}$ they reported 2 cases of Salmonella pancreatitis. Khan et al., ${ }^{2}$ reported cases of Salmonella pancreatitis with rhabdomyolysis and renal failure and also other studies quoting the same.

Our patient had a rare coexistence of acute hepatitis with coagulopathy and acute pancreatitis, which was reported earlier in one of the patients from Korea. ${ }^{10}$ Our patient also had delirium probably could be attributed as typhoid delirium (normal CT brain and CSF findings) and showed improvement only when he became a febrile irrespective of correction of metabolic derangements (hypocalcaemia and hypomagnesaemia). Therefore this may be the first reported case of a patient having all the rarer complications occurring together ever reported in literature and successfully treated conservatively and made a full recovery.

\section{Acknowledgements}

None.

\section{Conflict of interest}

The author declares no conflict of interest.

\section{References}

1. Sinha A, Sazawal S, Kumar R, et al. Typhoid fever in children aged less than 5 years. Lancet. 1999;354(9180):734-737.

2. Khan FY, Al-Ani A, AliHA. Typhoid rhabdo-myolysis with acute renal failure and acute pancreatitis: a case report and review of the literature. Int J Infect Dis. 2009;13(5):e282-e285.

3. Kadappu KK, Rao PV, Srinivas N, et al. Pancreatitis in enteric fever. Indian Journal of Gastroenterolog. 2003;21(1):32-33.

4. Osler W. Hepatic complications of typhoid fever. Johns Hopkins Hosp Rep. 1899;8:373-387.

5. Jagadish K, Patwari AK, Sarin SK, et al. Hepatic manifestations in typhoid fever. Indian Pediatr. 1994;31(7):807-811.

6. Pramoolsinsap C, Viranuvatti V. Salmonella hepatitis. J Gastroenterol Hepatol. 1998;13(7):745-750.

7. Karoli R, Fatima J, Chandra A, Singh G. Salmonella hepatitis: an uncommon complication of a common disease. J Fam Med Primary Care. 2012;1(2):160-162.

8. Zaki SA, Shanbag P. Co-existence of typhoid fever and hepatitis A. Ann Trop Med Public Health. 2014;5(5):551-552.

9. Balasubramanian S, Kaarthigeyan K, Srinivas S, et al. Serum ALT:LDH ratio in typhoid fever and acute viral hepatitis. Indian Pediatr. 2010;47(4):339-341.

10. Baek HS, Oh HT, Song SK, et al. A case of typhoid fever complicated by sensorineural hearing loss, acute pancreatitis and hepatitis. Korean $J$ infect Dis. 1994;29(1):57-61. 